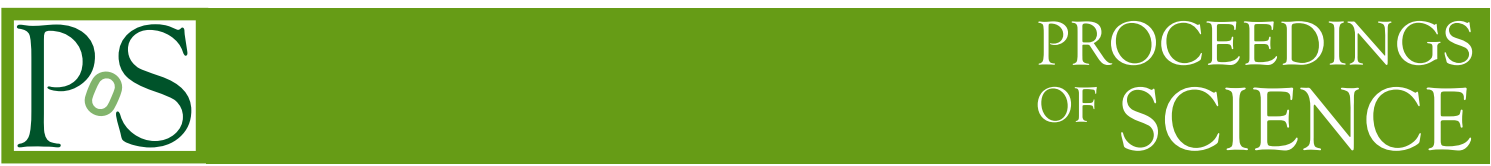

\title{
First Results from the LHCb Vertex Locator
}

\author{
Silvia Borghi* ${ }^{* \dagger}$ \\ Department of Physics and Astronomy, University of Glasgow, Glasgow, G12 8QQ, UK \\ E-mail:silvia.borghi@cern.ch
}

\begin{abstract}
LHCb is a dedicated experiment to study new physics in the decays of beauty and charm hadrons at the Large Hadron Collider (LHC) at CERN. The Vertex Locator (VELO) is the silicon detector surrounding the interaction point, and compared to the vertex detectors of the other LHC experiments, it is the closest LHC vertex detector to the beam interaction point, being located only 7 $\mathrm{mm}$ from the beam during normal operation. The detector operates in an extreme and highly nonuniform radiation environment. The VELO consists of two retractable detector halves with 21 silicon micro-strip tracking modules each. The VELO has been commissioned and successfully operated during the initial running period of the LHC. The preliminary operational results and detector performances are reported.
\end{abstract}

35th International Conference of High Energy Physics - ICHEP2010,

July 22-28, 2010

Paris France

\footnotetext{
* Speaker.

$\dagger$ On behalf of the LHCb VELO Group.
} 
LHCb [1] is a dedicated experiment to study New Physics in the decays of beauty and charm hadrons at the LHC at CERN. The Vertex Locator (VELO) is the silicon detector surrounding the interaction point. The VELO consists of two retractable detector halves with 21 silicon micro-strip modules each. A module is composed of two n+-on-n 300 micron thick half disc sensors with $\mathrm{R}$ and $\Phi$ micro-strip geometry, mounted on a carbon fibre support paddle. The detectors are operated in vacuum and a bi-phase $\mathrm{CO}_{2}$ cooling system is used. The LHC beam vacuum is separated from the detector vacuum by $300 \mu \mathrm{m}$ thick aluminium RF foils mounted on each half. In order to cover the full azimuthal acceptance and for alignment issues, the RF foils are corrugated in a way that allows the sensors of the two detector halves to overlap. During physics running conditions of the LHC machine, the sensors are operated at $7 \mathrm{~mm}$ from the beam. Since the required LHC aperture increases during beam injection and machine studies, the detector halves are then retracted by 30 $\mathrm{mm}$. The halves are inserted for each fill of the LHC once stable beams are obtained.

The VELO has been commissioned and successfully operated during the initial running period of the LHC. The detector has been time-aligned to the LHC beam within $2 \mathrm{~ns}$. After the time alignment the signal-to-noise ratio was evaluated: by a fit of a Landau convolved with a Gaussian the most probable value of the signal is extracted. The signal-over-noise ratio is measured to be 20:1. The cluster finding efficiency is evaluated by the extrapolation of each track to a sensor and looking for a cluster. The measured cluster finding efficiency, including the known bad strips, is 99.8\% and it is shown in fig. 1 as function of the module numbers. The spatial alignment of the modules obtained with early data has an accuracy of $4.4 \mu \mathrm{m}$. The variation of the alignment of one half with respect to the other one within fills is measured to be within $\pm 5 \mu \mathrm{m}$ for the horizontal translation (opening direction).

The small pitch and analogue readout, result in a best single hit precision of $4 \mu \mathrm{m}$ having been achieved at the optimal track angle. The preliminary primary vertex resolution with 25 tracks/vertex is determined to be $\sigma(x, y, z)=(16,15,90) \mu m$.

The VELO is fully operational and the detector performance are close to expectations.

\section{References}

[1] The LHCb Collaboration, The LHCb Detector at the LHC, J. Instrum. 3 (2008) S08005.
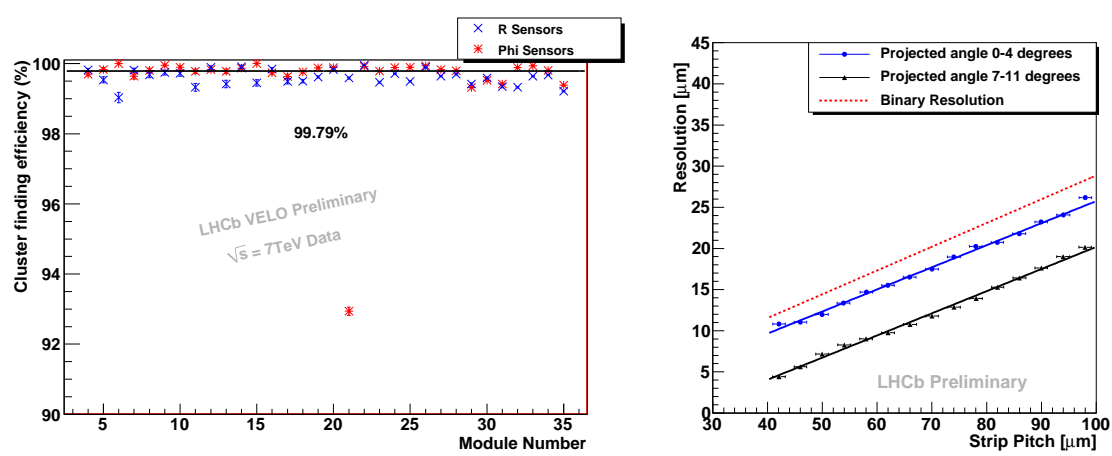

Figure 1: The left hand histogram shows the cluster finding efficiency for each sensors of the VELO. The right hand histogram shows the hit resolution versus the strip pitch in two projected angle regions. 